\title{
Fully Privacy-Preserving Distributed Optimization Based on Secret Sharing
}

This paper was downloaded from TechRxiv (https://www.techrxiv.org).

\section{LICENSE}

CC BY 4.0

SUBMISSION DATE / POSTED DATE

01-08-2021 / 03-08-2021

\section{CITATION}

Tian, Nianfeng; Guo, Qinglai; Sun, Hongbin; Zhou, Xin (2021): Fully Privacy-Preserving Distributed Optimization Based on Secret Sharing. TechRxiv. Preprint. https://doi.org/10.36227/techrxiv.15087774.v1

$\mathrm{DOI}$ 


\title{
Fully Privacy-Preserving Distributed Optimization Based on Secret Sharing
}

\author{
Nianfeng Tian, Student Member, IEEE, Qinglai Guo*, Senior Member, IEEE, \\ Hongbin Sun, Fellow, IEEE, Xin Zhou, Student Member, IEEE
}

\begin{abstract}
With the increasing development of smart grid, multiparty cooperative computation between several entities has become a typical characteristic of modern energy systems. Traditionally, data exchange among parties is inevitable, rendering how to complete multiparty collaborative optimization without exposing any private information a critical issue. This paper proposes a fully privacy-preserving distributed optimization framework based on secure multiparty computation (SMPC) secret sharing protocols. The framework decomposes the collaborative optimization problem into a master problem and several subproblems. The process of solving the master problem is executed in the SMPC framework via the secret sharing protocols among agents. The relationships of agents are completely equal, and there is no privileged agent or any third party. The process of solving subproblems is conducted by agents individually. Compared to the traditional distributed optimization framework, the proposed SMPC-based framework can fully preserve individual private information. Exchanged data among agents are encrypted and no private information disclosure is assured. Furthermore, the framework maintains a limited and acceptable increase in computational costs while guaranteeing optimality. Case studies are conducted to demonstrate the principle of secret sharing and verify the feasibility and scalability of the proposed methodology.

Index Terms-Secure Multi-Party Computation (SMPC), Privacy Preservation, Secret Sharing, Distributed Optimization.
\end{abstract}

\section{NOMENCLATURE}

\section{A. Indices/Sets}

$\begin{array}{ll}\mathcal{P} & \text { The set of agents } \\ P_{i} & \text { Agent } i \text { from set } \mathcal{P} \\ D_{i} & \text { The private data of agent } i \\ {\left[S_{i}\right]} & \text { The set of shares for } D_{i} \\ s_{i, k} & \text { The } k \text { th element from set }\left[S_{i}\right] \\ R & \text { The result for assignments } \\ {[S R]} & \text { The set of shares for } R \\ s r_{i, k} & \text { The } k \text { th element from set }[S R] \\ \omega & \text { The recombination vector }\end{array}$

\section{B. Parameters and Functions}

$f_{i}(\cdot) \quad$ The polynomial function of the initialization phase of agent $i$ in secret sharing

\begin{tabular}{|c|c|}
\hline$\Psi_{i}(\cdot)$ & $\begin{array}{l}\text { The mapping of the initialization phase of } \\
\text { agent } i \text { in secret sharing }\end{array}$ \\
\hline$\left[\Omega_{i}\right](\cdot)$ & $\begin{array}{l}\text { The mapping function of the computation } \\
\text { phase of agent } i \text { in secret sharing }\end{array}$ \\
\hline$\Omega(\cdot)$ & $\begin{array}{l}\text { The general form of the mapping function } \\
\text { of input } D \text { and output } R\end{array}$ \\
\hline$h(\cdot)$ & $\begin{array}{l}\text { The polynomial function for the resharing } \\
\text { process of secret multiplication }\end{array}$ \\
\hline$\Phi_{i}(\cdot)$ & $\begin{array}{l}\text { The mapping of the reconstruction phase in } \\
\text { secret sharing }\end{array}$ \\
\hline$R(\cdot)$ & $\begin{array}{l}\text { The Lagrange interpolation function for } \\
\text { reconstructing } R\end{array}$ \\
\hline$\oplus$ & Secure addition \\
\hline$\odot$ & Secure multiplication \\
\hline
\end{tabular}

\section{INTRODUCTION}

$\mathrm{W}$ ITH the increasing development of smart grid and energy internet [1][2], a massive number of scenarios characterized by cooperative optimization among multiple entities have emerged. The coordination among different entities, such as multiarea power grids, microgrids, and integrated electricity and gas systems, could offer additional flexibility to their operations and benefit the optimal resource allocation on a larger scale [3]. To enable cooperative computation, data exchange among entities is inevitable. However, the entities are usually independent stakeholders and intend to not expose confidential data to others, which is a barrier for further collaboration. Consequently, privacypreserving cooperative computation among multiple entities has become a critical issue.

One commonly considered approach is distributed optimization methods, such as the subgradient-based method, the alternating direction method of multipliers (ADMM), and its variants. Correspondently, the decomposition and coordination framework and the fully distributed framework have been developed. They have been widely studied and utilized in energy systems, such as power system unit commitment [4], microgrid operations [5], integrated energy system optimal schedules [6], etc. In [4], a modified subgradient method is utilized to decompose the model of the energy storage transportation scheduling in power systems and solve it in a decentralized manner. In [5], the ADMM is employed as a fully decentralized paradigm to solve the tie line scheduling among multiarea power grids without any coordinators. In [6], a standard ADMM approach with a coordinator and a consensus-based approach without any coordinators were developed to handle the synergistic 
operations of electricity and natural gas networks. Both systems only exchange their boundary states explicitly with each other in every iteration.

Although distributed optimization methods are advantageous in enhancing information confidentiality by limiting the amount and frequency of exchanged data, they could still result in potential problems due to the leakage of private information in the exchanged data [7]. Here, in the distributed optimization settings, we define privacy as the model-bounded confidential data, including the objective function, coefficients in constraints, subgradients, and optimal solutions.

Due to the privacy concerns in distributed optimization, existing works mainly consist of transformation-based methods [8], differential-privacy-based methods [9]-[11] and encryption-based methods [12]-[13]. First, in [8], private information in exchanged data is obscured by changing variables from the explicit form to a compact form. This transformation-based method highly relies on specific models and is weak in scalability, making it inappropriate for further applications in general scenarios. Second, regarding the differential privacy-based method, references [9]-[11] protect the sensitive data in optimization models by adding specifically designed noise to exchanged data. However, the added noise also unavoidably compromises the accuracy of optimization results, leading to a trade-off between privacy and accuracy. Additionally, differential privacy-based approaches may fail to converge to the accurate optimal solution even when no noise perturbation is added. These approaches are less promising in scenarios where accuracy is strictly required. Regarding the encryption-based method, references [12] and [13] introduced a partially homomorphic encryption system to the distributed framework of the ADMM. As the exchanged data are encrypted, private information in exchanged data can be well protected. However, in this method, computations on cipher-text data are restricted to simple arithmetic operations, such as addition. In addition, this method is only applicable to unconstrained optimization.

In this paper, Secure Multiparty Computation (SMPC) is utilized to construct the full privacy-preserving collaborative optimization framework. As a core technology in the field of information security, SMPC is a promising paradigm to tackle privacy issues in the collaborative optimization problem [14]. It is a theoretical framework proposed by Dr. Yao to solve the problem of cooperative computation between a group of participants who do not trust each other or a third party [15]. SMPC can ensure that the input information of all members participating in the calculation is not exposed. Furthermore, it can obtain accurate calculation results, which are guaranteed mathematically [16].

SMPC has been applied to the fields of secure voting [17], data sharing in the medical industry [18], blockchains [19], and data mining [20]. However, the considerable computational costs of conducting sophisticated assignments using SMPC protocols prevents further applications of SMPC. As the scale of the problem increases, the computational costs of SMPC will grow considerably. In view of both the advantage of SMPC in privacy protection and its drawback in computational burden, this paper proposes an SMPC-based distributed optimization framework to address the issues of privacy protection and the computational burden. In this framework, SMPC techniques preserve privacy, and the utilization of a distributed optimization framework helps reduce the computational complexity and communication burden. The main contributions are summarized as follows:

(i) The SMPC secret sharing protocols are introduced to establish a general privacy-preserving framework for multiparty collaborative optimization. The framework is essentially decentralized, and the relationships among agents are completely equal. There is no privileged agent or any third party. The exchanged data among agents are encrypted and no disclosure of the private information of any agent is assured.

(ii) To promote the performance of SMPC protocols in sophisticated multiparty collaborative optimization scenarios, an improved SMPC-based distributed optimization framework is proposed. The task flow of algorithms is based on the decomposition and coordination method. This method decomposes the collaborative optimization problem into a master problem and several subproblems. The process of solving the master problem uses the SMPC secret sharing protocol. Furthermore, the process of solving subproblems is conducted by agents individually.

(iii) Compared to the traditional distributed optimization framework, the proposed SMPC-based distributed optimization framework can fully preserve the private information of every agent without compromising the optimality of the method. Moreover, we fully use the advantage of the distributed optimization method to reduce the computational burden brought by the implementation of the SMPC framework. By specifically decomposing the optimization problem and simplifying the computation task in the master problem, the proposed method achieves a limited and acceptable increase in computational costs, which makes using the SMPC framework to handle sophisticated collaboration assignments practical.

The remainder of this paper is organized as follows: Section II introduces the basic SMPC theory and illustrates how the secret sharing scheme performs on generic assignments. In Section III, a general SMPC framework for multiparty collaborative optimization is proposed. Moreover, in Section IV, the fully privacy-preserving distributed optimization framework based on secret sharing is proposed. Then, Section $\mathrm{V}$ demonstrates the proposed methodology with case studies. Section 6 draws conclusions.

\section{II.SECURE MULTIPARTY COMPUTATION}

\section{A. Functionalities of Secure Multiparty Computation}

SMPC is a theoretical framework to solve the problem of cooperative computation between a group of participants who do not trust each other or a third party. It is characterized by functionalities including input privacy, computational correctness, and decentralization.

Input privacy: The research on secure multiparty computing focuses on the privacy security issues between all parties, which means that the secure multiparty computing process must 
ensure that the private input information of each party is independently processed without any disclosure.

Computational Correctness: All parties involved in multiparty computation can perform collaborative mathematical operations through an agreed SMPC protocol for a calculation task. As the computational process is finished, all parties will obtain their own correct data feedback.

Decentralization: In the SMPC framework, there is no privileged participant or third party, which means that the framework provides a fundamentally decentralized computing mode.

There are two current prevailing SMPC protocols [21], namely, protocols based on oblivious transfer and protocols based on homomorphic encryption. The $(t, n)$ threshold secret sharing scheme [22] is a typical cryptographic paradigm in protocols based on homomorphic encryption. The basic principle of $(\mathrm{t}, \mathrm{n})$ threshold secret sharing [23] is to randomly divide a secret $D$ into $n$ pieces of data $S_{1}, \cdots, S_{n}$ in such a way that the following holds.

(i) Knowledge of any $t-1$ or fewer pieces leaves $D$ completely undetermined.

(ii) The complete secret $D$ can be reconstructed from any combination of $t$ or more pieces.

In this paper, protocols based on Shamir's Secret Sharing (SSS) scheme, a mainstream threshold secret sharing paradigm based on Lagrange interpolation proposed by A. Shamir in 1979 [23], are utilized to explore the implementation of SMPC to power system optimization.

\section{B. The SMPC protocol based on SSS}

The SMPC protocol based on SSS enables generic computation on shared data, which is a special form in ciphertext. Fig. 1 gives an explicit illustration of the principles of SSS. Generally, each agent plays the roles of a client providing private input data and a server performing computation. The concrete algorithm is categorized into three phases, namely, the initialization phase $\Psi$, the computation phase $[\Omega]$, and the reconstruction phase $\Phi$.

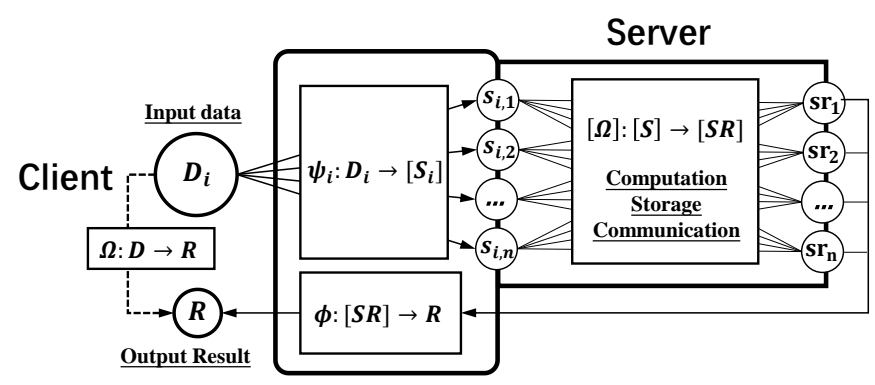

Fig. 1 Schematic diagram of the SMPC protocol based on SSS

First, in the initialization phase, each agent generates and exchanges shares of secrets (their input data) to ensure that each agent obtains all the input data in the form of shares. Then, in the computation phase, each agent takes the initialized shares as inputs to perform the SMPC protocols and obtain the results, which are also in the form of shares. Finally, in the reconstruction phase, sufficient numbers of shares in agents should be collected to reveal the real results. The following provides a detailed illustration:

1) Initialization Phase: $\Psi$

Suppose that agent $P_{i} \in \mathcal{P}$ keeps secret data $D_{i}$.

Step 1: $P_{i}$ generates elements $a_{i, 0}, a_{i, 1}, a_{i, 2}, \ldots, a_{i, t-1}$ randomly to construct polynomial $f_{i}(x)$ with the constant term $a_{i, 0}=D_{i}$.

$f_{i}(0)=D_{i}: f_{i}(x)=a_{i, 0}+\sum_{m=1}^{t-1} a_{i, m} x^{m}$

Step 2: $P_{i}$ calculates $\left[S_{i}\right]=\left\{s_{i, m} \mid s_{i, m}=f_{i}(m), m=1,2, \cdots, n\right\}$. Then, $P_{i}$ distributes $s_{i, m}(i \neq m)$ to $P_{m}$ through a secure communication channel. Let $\left[S_{i}\right]$ be a set of shares for data $D_{i}$, and $s_{i, m}$ is kept by $P_{m}$. The standard notation for shares is defined in (2), where $D_{i}$ is shared as $\left[S_{i}\right]$ through a polynomial $f_{i}(\cdot)$.

Notation : $D_{i} \sim f_{i} \rightarrow\left[S_{i}\right] \triangleq\left\{s_{i, 1}, s_{i, 2}, \cdots s_{i, n}\right\}$

In this way, each agent holds a copy of the secret data from all agents. An illustration of the methodology with a three-agent example is shown in Fig. 2.

(1) Generate Shares (2) Exchange Shares (3) Shares Initialized

(1)

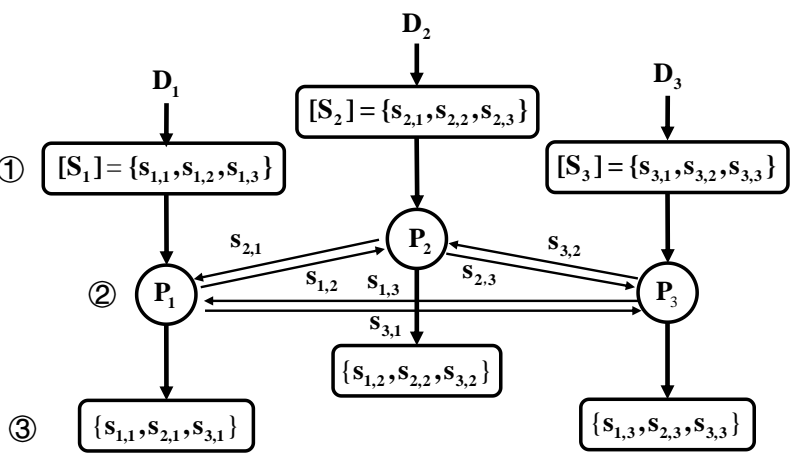

Fig. 2 The process of generating and allocating secret shares in the initialization phase: a three-agent example

\section{2) Computation Phase: $[\Omega]$}

It is proven that the computation task can be categorized into a polynomial mapping function $R=\Omega\left(D_{1}, D_{2}, \ldots, D_{n}\right)$, which can be described using an arithmetic circuit without any loss of generality. Therefore, the procedure in the computation phase consists of two basic operations, including secure addition $\oplus$ and secure multiplication $\odot$. Both can be built as SMPC gates to construct sophisticated functions.

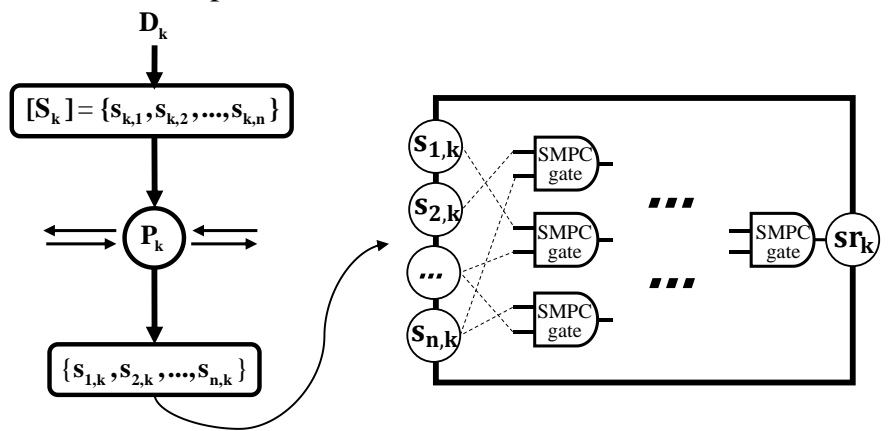

Fig. 3 SMPC gate builder of a server in the computation phase: each gate performs basic operations such as addition, multiplication, etc. 
The SMPC gate builder of a server in the computation phase is presented in Fig. 3. The input data of server $k$ is a set of initialized shares, where $s_{i, k}$ is one of the shares of secret $D_{i}$. In addition, the output $s r_{k}$ is the result derived from the input data through multiple layers of SMPC gates. The assignment for agent $k$ is to calculate $[\Omega]: S \rightarrow S R_{k}$. Note that the real value of the original objective $R$ can be revealed from $[S R] \triangleq\left\{S R_{1}, \ldots, S R_{n}\right\}$ as long as it holds that $[S R]$ is the secret share of $R$.

As defined in the initialization phase $\Psi$, the input $D_{i}$ of agent $P_{i}$ has been encrypted as a set of shares $\left[S_{i}\right] \triangleq\left\{s_{i, 1}, \ldots, s_{i, n}\right\}$ through a self-defined polynomial $f_{i}(x)$. When performing secure addition and secure multiplication on shares of secrets using SMPC gates [25], the key point is to ensure that the input and output of each gate are both in the form of shares.

\section{(a) Secure Addition: $\left[D_{i}+D_{j}\right]=\left[D_{i}\right] \oplus\left[D_{j}\right]$}

Note that the $(t, n)$ threshold scheme is naturally addictive homomorphic [14]. For each agent $P_{k}$, calculate $c_{k}=s_{i, k}+s_{j, k}$, and a new polynomial $f_{\mathrm{c}}(x)$ corresponding to $[c] \triangleq\left\{c_{k} \mid c_{k}=f_{c}\left(x_{k}\right), \forall k\right\}$ can be derived as (3).

$f_{\mathrm{c}}(x)=f_{i}(x)+f_{j}(x)=\left(D_{i}+D_{j}\right)+\sum_{m=1}^{t-1}\left(a_{i, m}+a_{j, m}\right) x^{m}$

Here, $f_{\mathrm{c}}(x)$ is a $t$-degree polynomial where $f_{\mathrm{c}}(0)=D_{i}+D_{j}$. Therefore, $[c]$ is exactly the secret share of the real value of the sum $D_{i}+D_{j}$. It holds that $D_{i}+D_{j} \sim f_{c} \rightarrow[c] \triangleq\left\{c_{1}, c_{2}, \cdots c_{n}\right\}$.

\section{(b) Secure Multiplication: $\left[D_{i} \cdot D_{j}\right]=\left[D_{i}\right] \odot\left[D_{j}\right]$}

Different from the processes in handling secure addition, secure multiplication cannot be simply accomplished by each agent $P_{k}$ calculating $s_{i, k} \cdot s_{j, k}$. Suppose that $d_{k}=s_{i, k} \cdot s_{j, k}$ and $f_{d}(x)$ is the corresponding polynomial of $[d]$, where $[d] \triangleq\left\{d_{k} \mid d_{k}=f_{d}\left(x_{k}\right), \forall k\right\}$. Therefore, $f_{d}(x)$ is written in the form of (4).

$$
\begin{aligned}
& f_{d}(x)=f_{i}(x) \cdot f_{j}(x)=\left(D_{i}+\sum_{m=1}^{t-1} a_{i, m} x^{m}\right)\left(D_{j}+\sum_{m=1}^{t-1} a_{j, m} x^{m}\right) \\
& =D_{i} \cdot D_{j}+\left(a_{i, 1} a_{j, 0}+a_{i, 0} a_{j, 1}\right) x+\cdots+\left(a_{i, t-1} a_{j, t-1}\right) x^{2 t-2}
\end{aligned}
$$

The degree of polynomial $f_{d}(x)$ is $2 t-1$, which is inconsistent with the form of shares initialized in (1). A resharing [24] of $d_{k}=s_{i, k} \cdot s_{j, k}$ is conducted to reduce the degree of polynomial correspondent to $D_{i} \cdot D_{j}$ back to $t-1$.

It is proven that for polynomial $f_{d}(x)$, there is a recombination vector $\omega=\left(\omega_{1}, \omega_{2}, \cdots, \omega_{n}\right)^{T}$ that satisfies $f_{d}(0)=\sum_{i=1}^{n} \omega_{i} f_{d}(i)$. In addition, $\omega$ is a publicly known vector taking the form of (5).

$$
\omega=\left(\begin{array}{ccccc}
1 & 1 & 1 & \cdots & 1 \\
1 & 2 & 2^{2} & \cdots & 2^{\mathrm{n}-1} \\
\vdots & \vdots & \vdots & \vdots & \vdots \\
1 & n & n^{2} & \cdots & n^{n-1}
\end{array}\right)^{-1}\left(\begin{array}{c}
1 \\
0 \\
\vdots \\
0
\end{array}\right)
$$

In the resharing process, $P_{k}$ generates a set of shares for $d_{k}$, which is in the form of (6). Then, $P_{k}$ multiplies each element in $\left[e_{k}\right]$ by $\omega_{i}$ and sends $\omega_{i} e_{k, i}$ to $P_{i}(i \neq k)$. Finally, the real value of $D_{i} \cdot D_{j}$ is shared in the form of (7).

$d_{k} \sim h_{k}(\cdot) \rightarrow\left[e_{k}\right] \triangleq\left\{e_{k, 1}, e_{k, 2}, \cdots e_{k, n}\right\}$

$D_{i} \cdot D_{j} \sim h(x) \rightarrow[g] \triangleq\left\{g_{1}, g_{2}, \cdots, g_{n}\right\}$

where $h(x)=\sum_{k=1}^{n} \omega_{k} h_{k}(x)$ and $g_{j}=\sum_{i=1}^{n} \omega_{i} e_{j}$

\section{3) Reconstruction Phase: $\phi$}

As presented in the computation phase, $[S R] \triangleq\left\{s r_{1}, \ldots s r_{n}\right\}$ is a set of shares of $R$, where $R$ is the real objective function and $s r_{i}$ is one of the shares of $R$ kept by $P_{i}$. [SR] and $R$ are related through a polynomial $R(x)$. Given that the input and output of each SMPC gate are all in the form of shares, the degree of $R(x)$ is $t$. Therefore, the real output $R$ can be obtained by gathering information $s r_{i}$ from any $t$ agents (without any loss of generality, suppose agents $P_{1}, P_{2}, \ldots, P_{t}$ ). The Lagrange interpolation formula is utilized to reconstruct $R(x)$ through tuples $\left(x_{1}, s r_{1}\right),\left(x_{2}, s r_{2}\right), \ldots,\left(x_{t}, s r_{t}\right)$. The real value of the objective function is obtained by $R=\left.R(x)\right|_{x=0}$.

$R(x)=\sum_{i=1}^{t} \frac{s r_{i}\left(x-x_{1}\right) \ldots\left(x-x_{i-1}\right)\left(x-x_{i+1}\right) \ldots\left(x-x_{t}\right)}{\left(x_{i}-x_{1}\right) \ldots\left(x_{i}-x_{i-1}\right)\left(x_{i}-x_{i+1}\right) \ldots\left(x_{i}-x_{t}\right)}$

In practical scenarios, the outputs may be known to specific agents and secret to others. Therefore, each agent is only authorized to recover the output that belongs to themselves.

\section{A GENERAL SMPC FRAMEWORK FOR MULTIPARTY COLLABORATIVE OPTIMIZATION}

Multiparty collaborative optimization is a typical type of problem in power system planning, operations and control, such as optimal power flow, secure-constrained unit commitment, etc. Several agents gather their information to obtain a global optimal solution that comprehensively benefits power system operations. Consider the general collaborative optimization problem in the form of Eq. (9) in which the objective and constraints contain both public parts and individual parts.

$$
\begin{array}{lll}
\min _{\mathrm{x}} & f^{\mathrm{pub}}\left(\mathrm{x}, \mathbf{D}^{\mathrm{obj}, \mathrm{pub}}\right)+\sum_{i=1}^{n} f_{i}\left(\mathrm{x}_{i}, \mathbf{D}_{i}^{\mathrm{obj}, \text { ind }}\right) & \\
\text { s.t. } & \mathrm{h}^{\mathrm{pub}}\left(\mathrm{x}, \mathbf{D}^{\mathrm{con}, \mathrm{pub}}\right)=0, \mathrm{x}=\left[\mathrm{x}_{1}^{\mathrm{T}}, \cdots, \mathrm{x}_{N}^{\mathrm{T}}\right]^{\mathrm{T}} & \text { public constraints } \\
& \mathrm{g}_{i}\left(\mathrm{x}_{i}, \mathbf{D}_{i}^{\mathrm{con}, \text { ind }}\right)=0, \forall i & \text { individual constraints }
\end{array}
$$

where the subscripts $/^{\text {con }}$ and $/^{\text {obj }}$ represent parameters or functions in the constraints and objective, respectively. The subscripts $/^{\text {pub }}$ and $f^{\text {ind }}$ represent the public and individual elements, respectively. $\mathbf{D}^{\text {obj, pub }}=\left[\left(D_{1}^{\text {obj, pub }}\right)^{\mathrm{T}}, \cdots,\left(\mathrm{D}_{n}^{\text {obj, pub }}\right)^{\mathrm{T}}\right]^{\mathrm{T}}$ is the vector of public parameters in the objective, and 
$\mathbf{D}^{\text {con, pub }}=\left[\left(\mathrm{D}_{1}^{\text {con, pub }}\right)^{\mathrm{T}}, \cdots,\left(\mathrm{D}_{n}^{\text {con, pub }}\right)^{\mathrm{T}}\right]^{\mathrm{T}}$ is the vector of public parameters in the public constraints.

Generally, D represents the private data of agents that serve as the boundary condition for collaborative optimization. $\mathrm{x}$ represents the output optimizers that each agent wishes to obtain by jointly solving the collaborative optimization. To avoid the potential privacy disclosure threat in gathering individual information, an SSS-based SMPC framework is proposed to tackle the collaborative optimization problem, as shown in Fig. 4. The main idea is to manage the input parameters, computing process, and output optimizers with the secret sharing scheme.

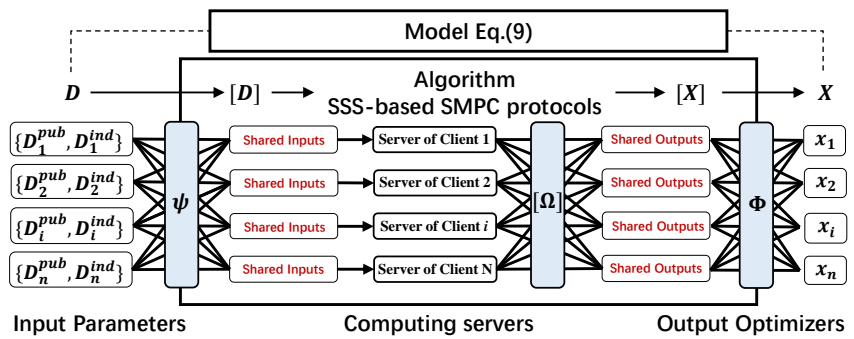

Fig. 4 The general SSS-based SMPC framework for multiparty collaborative optimization

First, the data of each agent are shared through the initialization phase $\Psi$ detailed in Section III.B. In this way, every agent obtains a group of shared pieces for all inputs. Then, the computation task for solving the collaborative optimization on shared inputs is conducted by executing an SSS-based SMPC protocol among servers of clients. In this protocol, an algorithm is conducted to map the shared inputs into shared outputs, and every step is composed of unit operations such as secure addition or secure multiplication. Finally, the real value of the optimizers of each client can be recovered by gathering a sufficient number of pieces of shared outputs and following the process of the reconstruction phase $\Phi$. Each client is only authorized to recover their own optimizer.

Note that no third party is needed in the SSS-based SMPC protocol, and every client maintains an equal relationship. Therefore, the proposed framework is essentially decentralized. Besides, according to the features of the secure computations in phase $[\Omega]$, each client is required to be capable of executing synchronous computation and conducting necessary communication with every other client.

Although the general SMPC framework for multiparty collaborative optimization performs well in privacy protection, the idea of transforming all the input data of clients into shares and solving the optimization on these shares will result in a massive increase in computational costs compared to solving the optimization problem in the traditional way. This is because in SMPC, any operation related to secret inputs must be conducted using secure computation protocols. Furthermore, secure computation is much more complex than traditional arithmetic operations. Therefore, the performance of SMPC heavily relies on the number of secret inputs. In this case, improving the performance of SMPC in computational and communicational complexity by reducing the number of private inputs in multiparty collaborative optimization problems is promising. The methodology is given in Section IV.

\section{Fully PRIVACY-PRESERVING DistribUTED OPTIMIZATION BASED ON SECRET SHARING}

Given the considerable increase in computational complexity in the general SMPC-based framework, several requirements should be followed to reduce the computational costs and obtain a desirable working performance. These requirements are listed below:

i). The entire model for collaborative optimization should be decomposed into the individual part and the public part, and the public part of the model should be simplified as much as possible.

ii). Due to the decomposition of the model, coordination among clients for convergence is indispensable. The decomposition method should facilitate coordination, which means that the communication burden among clients should be reduced as much as possible.

iii). The detailed operations of the algorithm utilized to build the SSS-based SMPC protocol should be simplified. Complex secure operations should be avoided as much as possible.

Above all, the decomposition and coordination (D\&C) method can accommodate these requirements well.

\section{A.The Improved SMPC Framework for Multiparty Collaborative Optimization Using the D\&C Method}

In the D\&C method, the collaborative optimization model of Eq. (9) is decoupled into a master problem and several subproblems. Each subproblem can be solved in an independent and parallel way. The Lagrangian function $L(\mathrm{x}, \Lambda)$ of Eq. (9) is defined as

$$
\begin{aligned}
& L(\mathrm{x}, \Lambda)=\sum_{i=1}^{n} f_{i}\left(\mathrm{x}_{i}, \mathbf{D}_{i}^{\text {obj, ind }}\right)+K(\mathrm{x}, \Lambda) \\
& K(\mathrm{x}, \Lambda)=f^{\text {pub }}\left(\mathrm{x}, \mathbf{D}^{\text {obj, pub }}\right)-\Lambda^{T} \cdot \mathrm{h}^{\text {pub }}\left(\mathrm{x}, \mathbf{D}^{\text {con, pub }}\right)
\end{aligned}
$$

The dual function is the objective of the dual problem, i.e., the supreme of the Lagrangian function over $\mathrm{x}$. By separating the coupling and decoupling terms in $\mathrm{h}^{\text {pub }}$ regarding decision variables $\mathrm{x}_{i}, K(\mathrm{x}, \Lambda)$ is rewritten as (11), where $K_{0}$ and $K_{i}$ represent the coupled part and the decoupled part in $K(\mathrm{x}, \Lambda)$, respectively.

$$
K(\mathrm{x}, \Lambda)=K_{0}(\mathrm{x}, \Lambda)+\sum_{i=1}^{n} K_{i}\left(\mathrm{x}_{i}, \Lambda\right)
$$

Since each client makes its own decision, the dual function can be decoupled into the sum of all clients' objectives. The subproblem is formed as

$$
\begin{array}{ll}
\min _{\mathrm{x}_{i}} & f_{i}\left(\mathrm{x}_{i}, \mathbf{D}_{i}^{\mathrm{obj}, \text { ind }}\right)+K_{i}\left(\mathrm{x}_{i}, \Lambda\right) \\
\text { s.t. } & \mathrm{g}_{i}\left(\mathrm{x}_{i}, \mathbf{D}_{i}^{\text {con, ind }}\right)=0
\end{array}
$$

According to the strong duality principle, the primal and dual problems are strictly equivalent. The dual problem can be solved iteratively by updating the multipliers, which is shown in (13).

$\max _{\Lambda} \min _{\mathrm{x}} K_{0}(\mathrm{x}, \Lambda)$

In the traditional $\mathrm{D} \& \mathrm{C}$ framework, a coordination layer is necessary for convergence. The layer communicates with all clients and obtains part of their private information to update the multipliers related to the public constraints. During this process, the coordination layer, serving as a third party, has access to the private information of every agent, which could 
Traditional decomposition and coordination framework

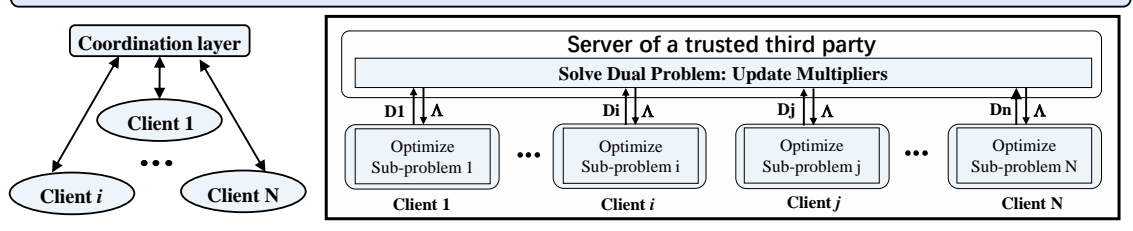

SMPC-based distributed optimization framework

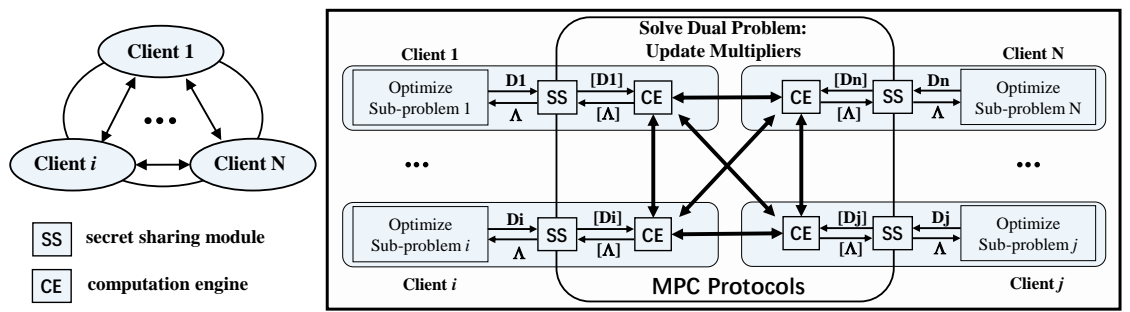

Fig. 5 Comparison of traditional decomposition and coordination framework and SMPC-based distributed optimization framework

result in severe privacy threats. Moreover, it is probably difficult to reach an agreement when choosing a generally trusted third party to play the role of coordination in a practical scenario, especially when the agents represent different stakeholders.

By introducing secret sharing into the decomposition and coordination framework, the computation task of the master problem is not conducted using a special coordination layer but by each client through the SMPC protocol based on secret sharing. The differences between these two frameworks are illustrated in Fig. 5. In this case, the collaborative optimization among clients can be realized in a decentralized manner. Since the exchanged data among clients are all in the form of shares, the privacy information of regional power grids is preserved.

TABLE I: ALGORITHM 1

\begin{tabular}{l}
\hline \hline Algorithm 1: Individual computation task \\
\hline Step 1: Agent $i$ collects the real value of multipliers $\Lambda$ \\
Step 2: Agent $i$ solves the subproblem Eq. (12) individually and obtains its \\
own optimizer $\mathrm{x}_{i}$ \\
Step 3: Agent $i$ initializes the set of shares $\left[\mathrm{x}_{i}\right]$ for optimizer $\mathrm{x}_{i}$ \\
following the instructions in phase $\Psi$ \\
>> Input: multipliers in real value $\Lambda$ \\
>> Output: Optimizers in shared form $\left[\mathrm{x}_{i}\right]$ \\
\hline \hline
\end{tabular}

TABLE II: ALGORITHM 2

\begin{tabular}{l}
\hline \hline Algorithm 2: Interactive computation task \\
\hline Step 1: Agents conduct SMPC protocols on shares $\left[\mathrm{x}_{i}\right]$ to solve the \\
master problem Eq. $(13)$ and then derive the multipliers in shared form \\
{$[\Lambda]$. Details follow the instructions in phase $[\Omega]$.} \\
>> Input: the set of shares $\left[\mathrm{x}_{i}\right]$ \\
>> Output: multipliers in shared form $[\Lambda]$ \\
\hline
\end{tabular}

TABLE III: ALGORITHM 3

\begin{tabular}{l}
\hline Algorithm 3: Reconstruction of the multiplier $\Lambda$ of public constraints \\
Step 1: Agents recover the real value of multipliers $\Lambda$ according to the \\
instructions in phase $\Phi$ \\
Step 2: Broadcast the real value of multipliers $\Lambda$ to every agent. \\
> Input: multipliers in shared form $[\Lambda]$ \\
>> Output: multipliers in real value $\Lambda$ \\
\hline
\end{tabular}

In this framework, each agent plays the role of a data provider and a computation server. The entire assignment for solving the collaborative optimization is decoupled into two parts, namely, individual computation tasks and interactive computation tasks. Individual computation tasks are conducted locally by each agent without interacting with other agents, and they refer to the subproblem (12). In addition, the interactive computation task refers to the master problem (13). Note that no coordination layer is needed. Instead, the interactive computation task is conducted through an SMPC protocol based on SSS. The entire assignment is accomplished by iteratively conducting the individual computation tasks and the interactive computation tasks until the optimizers converge. The convergence process of the improved SMPC framework based on the D\&C method is shown in Fig. 6; and the details for the three algorithms are given in TABLE I, TABLE II, and TABLE III.

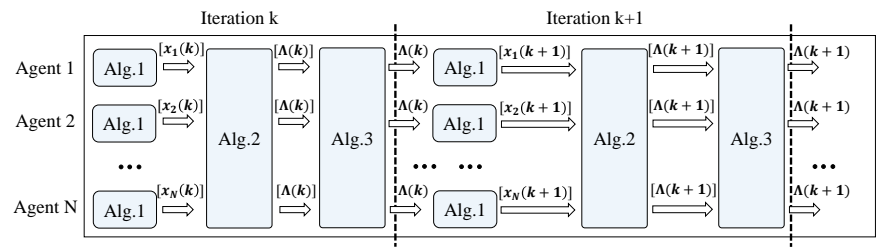

Fig. 6 The convergence process of the improved SMPC framework based on the D\&C method

\section{B. Discussion}

The features of the improved SMPC framework for multiparty collaborative optimization using the D\&C method depend on the proposed method's effectiveness and efficiency. The effectiveness is the feasibility of the proposed method in guaranteeing the input privacy and the correctness of the results. The efficiency is the proposed method's communication and computational complexity. Theoretical analysis is given below, and the demonstration is given in the case study.

\section{1) Effectiveness}

The key point when analyzing the security of the SMPC protocol based on secret sharing is how much each agent can infer of each other's information from the process of conducting protocols. 
As proposed in the improved SMPC framework using the D\&C method, the private data of each agent are divided into the individual part and the public part. The individual part is intrinsically secure since it is only involved in the individual computation task and is never externally released. The public part is involved in the interactive computation task whose inputs are all initialized shares. According to the methodology detailed in Section II once the shares of private input are initialized, the corresponding computational results are all in encrypted form, and the decryption can only be performed in a $(t, n)$ threshold manner. Therefore, the security of this protocol is fundamentally guaranteed. Moreover, the security level is tuned by $t$. The larger $t$ is, the more pieces of shares are needed to retrieve the real value of data.

Besides, communication among clients through secure channels is also a crucial component. It is assumed that secure point-to-point channels among clients could be assured. Therein, every client is fully connected with each other. In modern energy systems, the SMPC-based distributed optimization framework would be quite accustomed to assignments of collaboration among several large entities, such as power systems, transportation systems, gas systems, etc.

\section{2) Efficiency}

In terms of computational complexity, compared to the general SMPC framework, the improved SMPC framework using the $\mathrm{D} \& \mathrm{C}$ method decomposes the original multiparty collaborative optimization into the master problem and several subproblems. Therefore, SMPC protocols are only conducted to solve the master problem. Given that the scale of the master problem is much smaller than that of the original problem, the computational costs for conducting SMPC protocols in the improved framework has been greatly reduced compared to those in the general framework. Moreover, the subproblems can be solved in a parallel way since they are executed by clients individually, which further boosts the computation process. Besides, as the number of clients $n$ or the degree of polynomial $t$ increases, the computational costs for conducting SMPC protocols will increase accordingly.

In terms of communication complexity, communication among agents only occurs when conducting the interactive computation task. In this process, the amount and frequency of communication depend on the number of unit secure computations. Communication is spared when conducting secure addition. However, to conduct secure multiplication once, $n$ rounds of communication among agents are needed. Generally, communication complexity is positively correlated with the scale of the interactive computation task, such as the number of private inputs and the number of secure operations. Simplifying the model of the interactive computation task in practical scenarios would improve the performance of the SMPC framework.

\section{V.CASE STUDY}

\section{A. Scenario}

The multiarea economic dispatch (MAED) of power grids based on collaborative optimization comprehensively facilitates the safe and efficient operation of power systems. To ensure that each subgrid participates in this assignment fairly and reasonably, SMPC is a promising approach with which the MAED problem can be executed in a distributed manner while preserving the private information of subgrids.

\section{1) The Model of MAED}

The MAED problem can be modeled as a quadratic programming $(\mathrm{QP})$ problem, namely, DC optimal power flow, which is formed as (14).

$$
\begin{aligned}
\min _{p_{i}} & \sum_{n=1}^{N g} C_{n}\left(p_{n}\right) \\
\text { s.t. } & \sum_{n=1}^{N g} p_{n}=\sum_{n=1}^{N} L_{n} \\
& P_{n}^{\min } \leq p_{n} \leq P_{n}^{\max }, \forall n \\
& -F_{l}^{\max } \leq \sum_{n=1}^{N g} G_{l . n} p_{n}-\sum_{n=1}^{N} G_{l . n} L_{n} \leq F_{l}^{\max }, \quad \forall l
\end{aligned}
$$

where $C_{n}\left(p_{n}\right)=c_{2, i} p_{i}^{2}+c_{1, i} p_{i}, c_{1, i}$ and $c_{2, i}$ are the cost coefficients of the corresponding generation unit. $N$ and $N g$ are the number of all nodes and generation nodes, respectively. $p_{n}$ is a decision variable representing the output power of the generation unit in bus $n . P_{n}^{\min }, P_{n}^{\max }$ and $F_{l}^{\min }$ are the power limits on generators and lines, respectively. $G_{l, n}$ is the power transfer distribution factor between line $l$ and generation bus $n$.

\section{2) The SMPC framework for MAED using D\&C methods}

The MAED model is decoupled into several subproblems. Each subproblem can be solved in an independent and parallel way. By relaxing the public constraints on power balance and tie line power limits, the Lagrangian function of the MAED model is defined as

$$
\begin{aligned}
L(p, \lambda, \bar{\omega}, \underline{\omega})= & \sum_{n \in I_{G}} C_{n}\left(p_{n}\right)-\lambda^{T}\left(\sum_{n \in I_{G}} p_{n}-\sum_{n \in I_{G}} D_{n}\right)-\bar{\omega}^{T}\left(F_{l}^{\max }-\sum_{n \in I_{G}} G_{k} p_{n}\right) \\
& -\underline{\omega}^{T}\left(\sum_{n \in I_{G}} G_{k} p_{n}-F_{l}^{\min }\right) \\
= & \sum_{n \in I_{G}} L_{n}(p, \lambda, \bar{\omega}, \underline{\omega})+K(\lambda, \bar{\omega}, \underline{\omega})
\end{aligned}
$$

where

$$
\begin{aligned}
& L_{n}(p, \lambda, \bar{\omega}, \underline{\omega})=C_{n}\left(p_{n}\right)-\lambda^{T} p_{n}+\bar{\omega}^{T} G_{k, n} p_{n}-\underline{\omega}^{T} G_{k, n} p_{n} \\
& K(\lambda, \bar{\omega}, \underline{\omega})=\lambda^{T} \sum_{n \in I_{G}} D_{n}-\bar{\omega}^{T} F_{l}^{\max }+\underline{\omega}^{T} F_{l}^{\min }
\end{aligned}
$$

$\lambda, \bar{\omega}, \underline{\omega}$ refer to the multipliers of the constraints on the power balance, tie line flow upper limit and lower limit, respectively. The dual function is the objective of the dual problem, i.e., the supreme of the Lagrangian function over the generation power. $\max _{\lambda, \bar{\omega}, \underline{\omega}} \min _{p} L_{n}(p, \lambda, \bar{\omega}, \underline{\omega})$

$$
\begin{aligned}
\min _{p_{n}} L_{n}\left(p_{n}, \lambda, \bar{\omega}, \underline{\omega}\right) & =C_{n}\left(p_{n}\right)-\lambda^{T} p_{n}+\bar{\omega}^{T} G_{k, n} p_{n}-\underline{\omega}^{T} G_{k, n} p_{n} \\
& =c_{n} p_{n}-\lambda^{T} p_{n}+\bar{\omega}^{T} G_{k, n} p_{n}-\underline{\omega}^{T} G_{k, n} p_{n} \\
& =\left(c_{n}-\lambda^{T}+\bar{\omega}^{T} G_{k, n}-\underline{\omega}^{T} G_{k, n}\right) p_{n}
\end{aligned}
$$$$
\text { s.t. } P_{n}^{\min } \leq p_{n} \leq P_{n}^{\max }
$$

Since each subgrid makes its own decision, the dual function can be decoupled into the sum of all generators' objectives. Therefore, the subproblem of each subgrid is given in Eq. (18). In the coordination layer, the master problem is formed as Eq. 
(17), which can be solved through the subgradient method. In this case, by applying the subgradient method to Eq. (17), the updating of multipliers follows (19). According to the strong duality principle, the primal and dual problems are strictly equivalent. The dual problem can be solved iteratively by updating the multipliers. The details of applying the proposed SSS-based SMPC framework to the MAED problem are shown in Fig. 7.

$$
\begin{aligned}
& d \lambda(k)=\sum_{n \in I_{G}} L_{n}-\sum_{n \in I_{G}} p_{n} \\
& d \bar{\omega}(k)=\left\{\begin{array}{cc}
0 & \text { if } \bar{\omega}(k)=0 \text { and } \sum_{n \in I_{G}} G_{k} p_{n} \leq F_{k}^{\max } \\
\sum_{n \in I_{G}} G_{k} p_{n}-F_{k}^{\max } & \text { otherwise }
\end{array}\right. \\
& d \underline{\omega}(k)=\left\{\begin{array}{cc}
0 & \text { if } \underline{\omega}(k)=0 \text { and } \sum_{n \in I_{G}} G_{k} p_{n} \geq F_{l}^{\min } \\
F_{l}^{\min }-\sum_{n \in I_{G}} G_{k} p_{n} & \text { otherwise }
\end{array}\right.
\end{aligned}
$$

In Fig. 7, the entire assignment of the MAED problem is conducted by iteratively solving the master problem and subproblems. Therefore, within each iteration, there are two tasks. First, the individual model is executed within each subgrid to update the power of generators $p_{n}(k)$. Second, the SSS-based SMPC protocols executed by the interactive communication and computation of all subgrids to solve the master problem and update the Lagrangian multipliers of public constraints $\lambda(k), \bar{\omega}(k), \underline{\omega}(k)$.

As defined in Section IV, the subproblems are solved in plaintext by each subgrid using traditional QP algorithms. Furthermore, the master problem is solved by the SSS-based SMPC protocols in ciphertext, where secure computation is performed on the shares of secret inputs. Therefore, the exchanged data among subgrids for solving the master problem, including the demand $D_{i}$, the power transfer distribution factors $G_{l, i}$ and the power of generators $p_{i}(k)$, are all initialized in the form of shares before being exchanged. Accordingly, the updated multipliers derived from solving the master problem are in the form of shares and should be recovered and then broadcast to every subgrid.

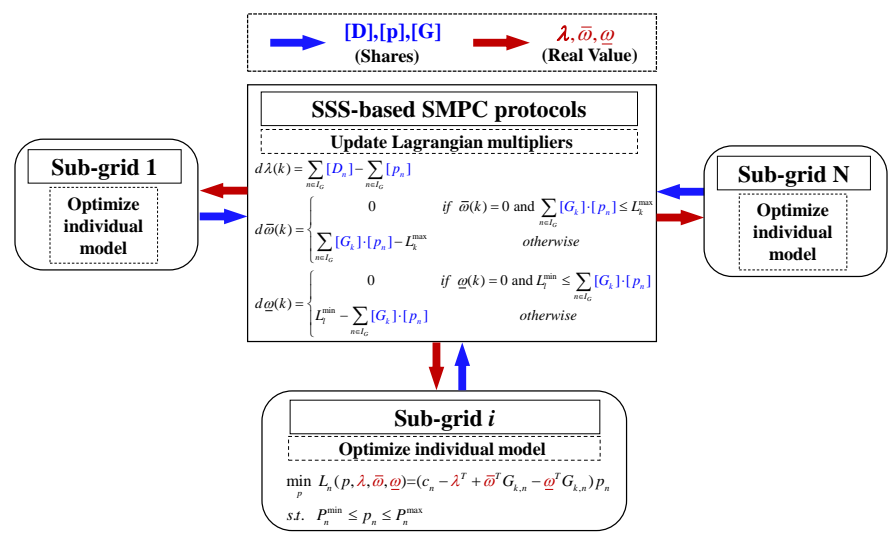

Fig. 7 The SSS-based fully privacy-preserving distributed framework of MAED

\section{B. Simulation Results}

The following case studies are both run in $M P y C$ [26], which is an SMPC platform based on Shamir's secret sharing scheme illustrated in Section II. $M P y C$ is included in Python as a package. Each agent is represented by an async function.

\section{1) Simple case}

To explicitly demonstrate how the SSS scheme works, we designed a 3-bus test system, shown in Fig. 8, where each bus represent a subgrid. The MAED model is performed considering public constraints on both power balance and tie line flow capacities. Every bus plays the role of an individual operator. Within each iteration, the operator collaborates with others to update the multipliers related to public constraints by exchanging necessary information in the form of shares. The unit power of each area is optimized after obtaining the updated multipliers. The parameters and optimal schedule of the generators and loads of each area are given in TABLE IV and TABLE V.

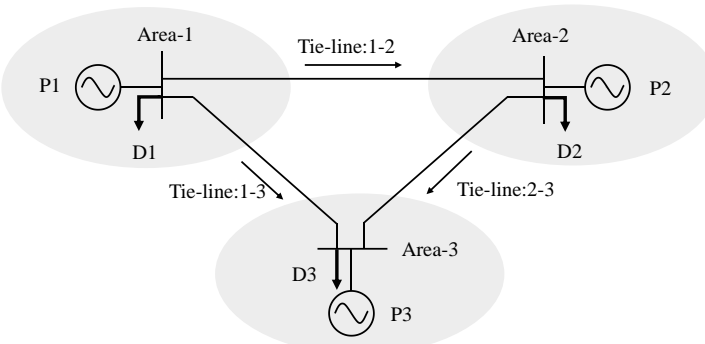

Fig. 8 The 3-bus test system

TABLE IV: PARAMETERS OF GENERATORS AND LOADS IN EACH AREA

\begin{tabular}{lllll}
\hline \hline & Notation & \multicolumn{1}{c}{ Area 1 } & \multicolumn{1}{c}{ Area 2 } & \multicolumn{1}{c}{ Area 3 } \\
\hline Load & $D$ & $1 \mathrm{MW}$ & $2 \mathrm{MW}$ & $3 \mathrm{MW}$ \\
Cost & $c_{1}$ & $3 \$ / \mathrm{MWh}$ & $2 \$ / \mathrm{MWh}$ & $1 \$ / \mathrm{MWh}$ \\
parameter & $c_{2}$ & $1 \$ / \mathrm{MWh}^{2}$ & $1 \$ / \mathrm{MWh}^{2}$ & $1 \$ / \mathrm{MWh}^{2}$ \\
& $P^{\max }$ & $3 \mathrm{MW}$ & $3 \mathrm{MW}$ & $3 \mathrm{MW}$ \\
Power limit & $P^{\min }$ & 0 & 0 & 0 \\
& & & & \\
\hline \hline
\end{tabular}

TABLE V: THE OPTIMAL SCHEDULE OF GENERATORS AND TIE LINES

\begin{tabular}{llllll}
\hline \hline D1 & 1 MW & P1 & 1 MW & Tie line:1-2 & $-2 / 3 \mathrm{MW}$ \\
D2 & $2 \mathrm{MW}$ & P2 & 2 MW & Tie line:1-3 & $-4 / 3 \mathrm{MW}$ \\
D3 & $3 \mathrm{MW}$ & P3 & 3 MW & Tie line:2-3 & $-2 / 3 \mathrm{MW}$ \\
\hline \hline
\end{tabular}

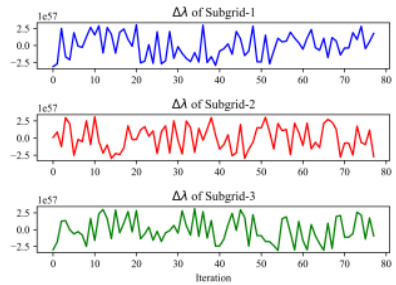

(a)

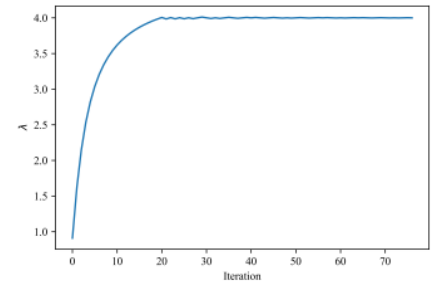

(b)
Fig. 9 the convergence performance of multipliers: (a) the shared value of $\Delta \lambda$ among subgrids, and (b) the real value of $\lambda$

The observations in Fig. 9 and Fig. 10 show that the secret sharing value of $\Delta \lambda$ and the optimized unit output at each step in each subgrid vary and are totally different from the curve of the real value. Moreover, the curves in secret sharing are randomly distributed and contain no information about the convergence process. However, the real value retrieved by the 
SMPC-based scheme shows steady convergence. Therefore, the proposed methodology can fundamentally protect the private information of subgrids at each iteration.

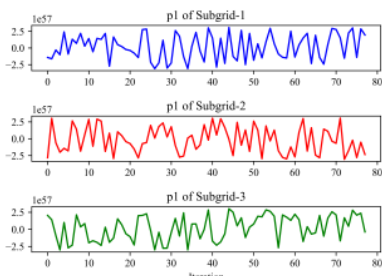

(a)

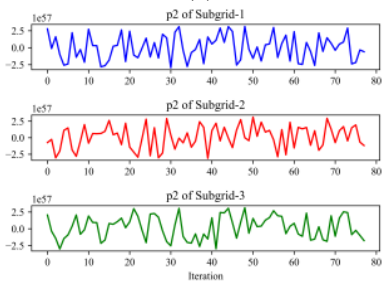

(c)

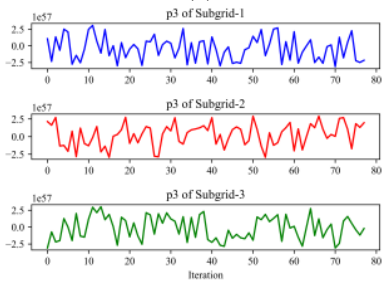

(e)

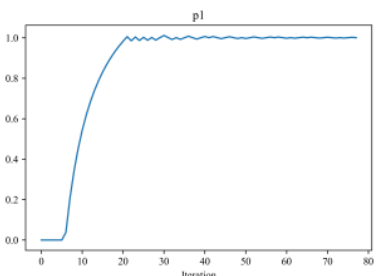

(b)

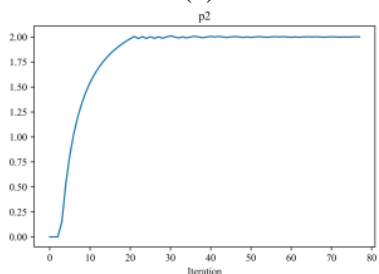

(d)

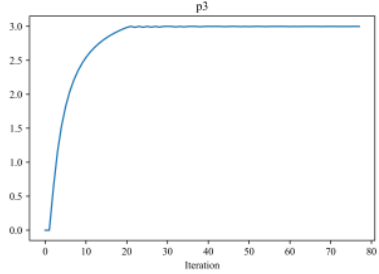

(f)
Fig. 10 The convergence performance of the unit output: (a, c, and e) show the secret sharing value of unit output $p$ of each subgrid, and (b, d, and f) show the real value of $p$ of each subgrid

\section{2) Large case}

To verify the feasibility and effectiveness of the proposed method, a case study is conducted on the IEEE-118 bus system. The total power system is divided into 3 subgrids, as shown in Fig. 11. Each subgrid is connected to the adjacent subgrids through tie lines.
Two aspects of the simulation results, the effectiveness and efficiency, are shown in TABLE VI. First, in terms of effectiveness, the optimal objective of M1 and M2 remains the same at \$10385.7. In addition, the optimal solution of each subgrid (with optimal generation output as the blue bar and power limits as the red dotted line) remains the same between M1 and M2, which demonstrates that the application and execution of SMPC protocols does not interfere with the accuracy of the results. Moreover, the convergence process of the residual, as well as the number of iterations, remains the same, which shows a beneficial feature that solving the optimization using SMPC would not change the convergence process. Finally, the optimality of the MAED problem is fundamentally guaranteed.

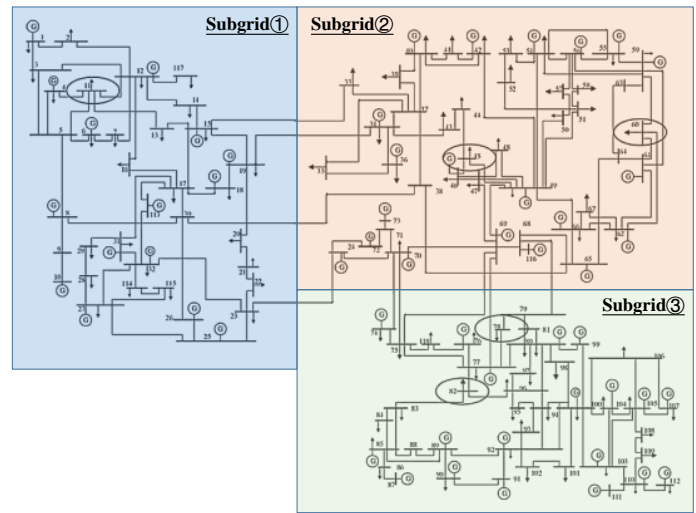

Fig. 11 The structure and partition of subgrids in the IEEE 118 bus test system

Second, compared to traditional method M1, SMPC-based M2 shows a minor increase in solution time from $3.125 \mathrm{~s}$ to $3.375 \mathrm{~s}$. For such a large system with many constraints and decision variables, the implementation of the SMPC-based distributed optimization method maintains a limited and acceptable increase in computational costs. This is because the assignment that needs to be calculated through SMPC protocols during each iteration is limited to several steps of the dot product, which is given in (19). Therefore, the SMPC framework for solving the MAED problem is scalable.

TABLE VI: COMPARISON OF THE SCALABILITY PERFORMANCE BETWEEN THE TRADITIONAL LR METHOD AND SMPC-MODIFIED LR METHOD

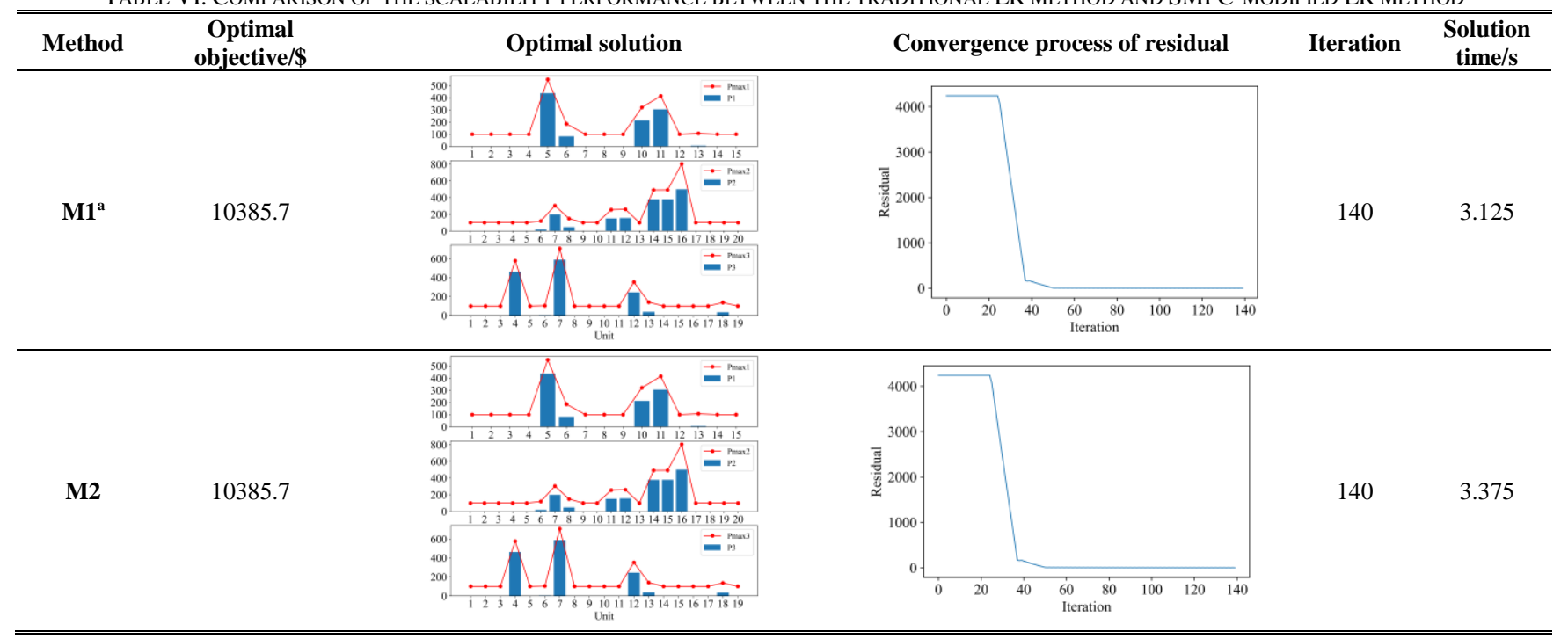

\footnotetext{
${ }^{\mathrm{a}}$ M1 refers to the traditional Lagrangian relaxation method, and M2 refers to the SMPC-based distributed optimization method
} 


\section{CONCLUSION}

In this paper, a fully privacy-preserving distributed optimization framework based on SMPC secret sharing protocols is designed and elaborated to handle multiparty collaborative optimization in power systems. The framework is performed using Lagrangian relaxation algorithms, and the role of the coordination layer is illuminated. In this way, the proposed framework is essentially decentralized, and each agent maintains an equal relationship. Multiparty collaborative optimization is iteratively calculated. In each iteration, every agent is assigned to optimize their subproblem, cooperates with each other to solve the master problem and updates the multipliers through the SMPC protocols. The exchanged data among agents are in the form of shares. The method guarantees that the real data are unable to be revealed unless a sufficient number of pieces of shares are collected. The case study based on multiarea economic dispatch with test systems on small and large scales verifies the optimality and scalability of the proposed framework. Using secret sharing techniques, the SMPC-based distributed optimization framework can provide an accurate global optimal solution and prevent the disclosure of individual private information. Moreover, the increase in computation time brought on by the implementation of SMPC protocols is minor and acceptable.

In our proposed SMPC-based distributed optimization framework, the subgradient method based on Lagrangian relaxation is utilized. Although it accommodates the requirement of reducing the computational complexity of SMPC protocols, it achieves less desirable convergence performance due to its susceptibility to the initial conditions. A better decomposition and coordination method that enhances the computational performance of the SMPC framework will be investigated in future work. Hopefully, the proposed methodology can provide new insights for the operation of modern energy systems with multiparty collaboration scenarios.

\section{REFERENCES}

[1] Wu J, Yan J, Jia H, Hatziargyriou N, Djilali N, Sun H. Integrated energy systems. Applied Energy 2016;167:155-7.

[2] Li G, Zhang R, Jiang T, Chen H, Bai L, Cui H, et al. Optimal dispatch strategy for integrated energy systems with cchp and wind power. Applied Energy 2017;192:408-19.

[3] Sun H, Guo Q, Zhang B, et al. Integrated energy management system: concept, design, and demonstration in China. IEEE Electrif Mag 2018;6(2):42-50.

[4] Yingyun, Sun, Zuyi, Li, Wei, \& Tian, et al. (2016). A lagrangian decomposition approach to energy storage transportation scheduling in power systems. IEEE Transactions on Power Systems, 31(6), 4348-4356.

[5] Li, Z. , Wu, W. , Zeng, B., Shahidehpour, M. , \& Zhang, B. . (2016). Decentralized contingency-constrained tie-line scheduling for multi-area power grids. IEEE Transactions on Power Systems, 354-367.

[6] Wen, Y., Qu, X. , Li, W. , Liu, X. , \& Ye, X. . (2017). Synergistic operation of electricity and natural gas networks via admm. IEEE Transactions on Smart Grid, 1-1.

[7] Zhang, X., Khalili, M. M. , \& Liu, M. . (2018). Improving the Privacy and Accuracy of ADMM-Based Distributed Algorithms. International Conference on Machine Learning.
[8] Zhang Y., Liu X., Yan Z. and Zhang P. (2020). DecompositionCoordination Based Optimization for PV-BESS-CHP Integrated Energy Systems. Trnasaction of China Electrotechnical society, vol.35, no. 11, 2372-2386

[9] Z. Huang, S. Mitra, and N. Vaidya, "Differentially private distributed optimization," in Proc. Int. Conf. Distrib. Comput. Netw., 2015,Art. no. 4.

[10] S. Han, U. Topcu, and G. J. Pappas, "Differentially private distributed constrained optimization," IEEE Trans. Autom. Control, vol. 62, no. 1, pp. 50-64, Jan. 2017.

[11] E. Nozari, P. Tallapragada, and J. Cortés, "Differentially private distributed convex optimization via objective perturbation," IEEE Trans.Control Netw. Syst., vol. 5, no. 1, pp. 395-408, Mar. 2018.

[12] Zhang, C. , \& Wang, Y. . (2017). Privacy-preserving decentralized optimization based on admm. IEEE Transactions on Information Forensics and Security.

[13] Zhang, C. , \& Wang, Y. . (2019). Enabling privacy-preservation in decentralized optimization. IEEE Transactions on Control of Network Systems, 6(2), 679-689.

[14] Cramer, R., Daza, V. , Gracia, I. , Urroz, J. J. , \& C Padró. (2005). On codes, matroids and secure multi-party computation from linear secret. Annual International Cryptology Conference. Springer Berlin Heidelberg.

[15] Yao, A. C. . (1982). Protocols for secure computation. Foundations of Computer Science, 1982. SFCS '08. 23rd Annual Symposium on. IEEE.

[16] Zhao C., Zhao S., Zhao M., Chen Z., Gao C., Li H. \& Tan Y. (2019). Secure Multi-Party Computation: Theory, practice and applications. Information Sciences, 476, 357-372.

[17] Dang-Awan, R. , Piscos, J. A. , \& Chua, R. B. . (2018). Using Sharemind as a Tool to Develop an Internet Voting System with Secure Multiparty Computation. 2018 9th International Conference on Information, Intelligence, Systems and Applications (IISA). IEEE Computer Society.

[18] Zyskind, G. , Nathan, O. , \& Pentland, A. . (2015). Enigma: decentralized computation platform with guaranteed privacy. Computer Science.

[19] Halevi, T. , Benhamouda, F. , Caro, A. D. , Halevi, S. , Jutla, C. , \& Y Manevich, et al. (2020). Initial Public Offering (IPO) on Permissioned Blockchain Using Secure Multiparty Computation. 2019 IEEE International Conference on Blockchain (Blockchain). IEEE.

[20] Smahi, A., Qi, X., Hu, X. , Sulemana, N. , \& Guizani, M. . (2020). A blockchainized privacy-preserving support vector machine classification on mobile crowd sensed data. Pervasive and Mobile Computing, 66, 101195.

[21] Luo W. (2005). Secure Multiparty Computation Theory and Application, Guizhou University.

[22] Karnin, E. D. , Greene, J. W. , \& Hellman, M. E. . (1983). On secret sharing systems. IEEE Trans on It, 29(1), 35-41.

[23] "How to share a secret." Communications of the Acm (1979).

[24] Goldwassert, S. , \& Wigdemon, A. . (1988). Completeness Theorems for Non-Crypto- graphic Fault-Tolerant Distributed Computation.

[25] Cramer, R. , Da Mgrd, I. B. , \& Nielsen, J. B. . (2015). Secure Multiparty Computation and Secret Sharing. Cambridge University Press.

[26] MPyC, Secure Multiparty Computation in Python. [Online]. Available: https://github.com/lschoe/mpyc. 